\title{
EDITORIAL
}

\section{Will all ARDS patients be receiving mechanical ventilation in 2035? We are not sure}

\author{
Michael Quintel, Tommaso Tonetti and Luciano Gattinoni*
}

(C) 2016 Springer-Verlag Berlin Heidelberg and ESICM

Prediction of the future is a risky game: who really knows if and how mechanical ventilation will be applied in 20 years from now? Before entering this opaque, foggy territory, the fundamental question to be answered in this context is: do we expect human physiology to change over the next 20 years? With great probability, unless extraordinary and unexpected evolutionary miracles occur, the answer will be "no". We may consequently try to rationally approach this question while considering on one side physiology and on the other expected or even unexpected technical improvements.

During the past few years, as a reaction to the high volumes and pressures applied during mechanical ventilation, the term "protective ventilation" [1-3] has been introduced. In our opinion, this expression is, however, deeply misleading, as the mechanical ventilation does not protect the lung from the development of the acute respiratory distress syndrome (ARDS), but simply aims to protect it from avoidable damage due to harmful ventilatory settings. The goal of mechanical ventilation is in fact not an imaginary and impossible "protection of the lung", but simply to buy time for the lung to heal, while maintaining a viable gas exchange. Therefore, it is worth noting that mechanical ventilation acts on $\mathrm{CO}_{2}$ clearance and $\mathrm{O}_{2}$ intake through different mechanisms.

- $\mathrm{CO}_{2}$ clearance. To eliminate the $\mathrm{CO}_{2}$, the lungs must be ventilated. As in ARDS, the "baby lung" $[4,5]$ may be extremely small, and each tidal volume entering the baby lung will dilute the $\mathrm{CO}_{2}$ to a far greater

${ }^{*}$ Correspondence: gattinoniluciano@gmail.com

Department of Anesthesiology, Emergency and Intensive Care Medicine, University of Göttingen, Robert-Koch-Straße 40, 37075 Göttingen,

Germany

For contrasting viewpoints, please go to doi:10.1007/s00134-016-4461-x and doi:10.1007/s00134-016-4487-0. extent than with a normal FRC. The expired end-tidal $\mathrm{CO}_{2}$ will be proportionally lower and measured as increased alveolar dead space. The $\mathrm{CO}_{2}$ retention further stimulates the ventilatory drive, and the high ventilation requirements to maintain a normal $\mathrm{PaCO}_{2}$ in the presence of a baby lung cannot be maintained by the respiratory muscles. In this scenario, the primary function of mechanical ventilation is to substitute the power of the respiratory muscles by the power of the ventilator in order to provide $\mathrm{CO}_{2}$ clearance. Obviously, accepting hypercapnia would reduce the need for ventilator support without abolishing it [6].

- $\mathrm{O}_{2}$ intake. While the baby lung is characterized by elevated ventilation-perfusion ratios, the perfusion of the remaining collapsed/consolidated lung causes, on the post alveolar side of the lung, mixing of "unaltered" venous blood with the blood fraction coming out from the baby lung [7]. Increasing the $\mathrm{FiO}_{2}$ increases the oxygenation of the blood perfusing the baby lung, but if the fraction perfusing the non-aerated lung is greater than $35 \%$, the arterial $\mathrm{PaO}_{2}$ cannot reach the normal values of $100 \mathrm{mmHg}$, even if the baby lung is ventilated with $100 \%$ of oxygen. This condition has been called "refractory hypoxemia" and it should still be called that nowadays. Mechanical ventilation may interfere with oxygenation (for a given alveolar $\mathrm{PACO}_{2}$ ) via two main mechanisms:

- It may provide accurate concentration of oxygen in the inspired gases.

- It recruits at least parts of the collapsed alveoli during the inspiratory phase.

Note that the positive end-expiratory pressure is not part of ventilation per se, but is associated with it via valve settings chosen during mechanical ventilation. In 
fact, continuous positive pressure may be easily provided, even with spontaneous breathing.

From what we have discussed above, the bases of the theoretical limits for a safe mechanical ventilation should be clear:

(a) Mechanical ventilation in ARDS is needed for $\mathrm{CO}_{2}$ clearance. Accepting a $\mathrm{PaCO}_{2}$ double that of normal allows the halving of the alveolar ventilation required. However, even accepting high $\mathrm{PaCO}_{2}$, the "tidal volume/baby lung" ratio (associated with lung inhomogeneities) and the presence of "stress raisers" may certainly lead to unsafe mechanical ventilation [8]. The identification of an exact threshold above which mechanical ventilation would be associated with an unacceptable risk of further damaging the lungs would allow a rational use of mechanical ventilation and an alternative use of extracorporeal lung support.

(b) The main requirements for oxygenation are elevated $\mathrm{FiO}_{2}$ and continuous positive pressure, for which mechanical ventilation is not strictly necessary.

It is important to emphasize that mechanical ventilation utilizes the residual ventilatable lung present in ARDS. Like furosemide, it works in a partially functioning organ: to substitute renal function renal replacement is required, to substitute the alveolar capillary function, artificial lungs are needed [9].

After stating this, any speculation on the future of mechanical ventilation will depend on our ability in 20 years from now to clearly identify, diagnose and quantify the triggers of ventilator-induced lung injury (VILI), i.e., the interaction between the mechanical power delivered by the ventilator and its distribution in a small and inhomogeneous lung. Clearly, in the absence of futuristic solutions like artificial red blood cells for oxygen transport or physico-chemical manipulations of blood $\mathrm{CO}_{2}$, even by 2035 the treatment alternative for ARDS will be between mechanical ventilation and various forms of extracorporeal support or their combination. A clear benefit and risk analysis seems mandatory before whatever type of respiratory support in the clinical setting is recommended. Clearly, in mild ARDS, supportive measures such as high-flow oxygen or non-invasive ventilation are easily feasible; these patients in fact present a large amount of ventilatable and homogenous lung. It seems likely that mechanical ventilation in these patients carries a comparably low risk. At the other end of the spectrum, in severe ARDS, characterized by "refractory hypoxemia" and shunt fractions greater than $35 \%$ [7], the risks of mechanical ventilation must be balanced with the risks of extracorporeal support [9]. The former is primarily represented by the unavoidable VILI, the latter in worst cases by cerebral hemorrhage. Obviously, if the technology around the artificial lung improves in the near future such that the need for systemic anticoagulation would be avoided or at least considerably reduced, the argument in favor of partial or total extracorporeal support will greatly increase. Finally, it must be remembered that, in early severe ARDS, even during artificial lung support, spontaneous breathing due to the increased respiratory drive may be more dangerous than any form of mechanical ventilation [10].

In conclusion, we believe that the improvements in the application of mechanical ventilation will be linked to a better characterization of the diseased lung of each individual patient, while the improvement in the application of extracorporeal support will be linked to a solution of anticoagulation-dependent problems.

\section{Compliance with ethical standards}

Conflicts of interest

On behalf of all authors, the corresponding author states that there is no conflict of interest.

Received: 14 July 2016 Accepted: 18 July 2016

Published online: 11 August 2016

\section{References}

1. Amato M et al (1996) Improved survival in ARDS: beneficial effects of a lung protective strategy (abstract). Am J Respir Crit Care Med 153(4):A531

2. Amato MB et al (1998) Effect of a protective-ventilation strategy on mortality in the acute respiratory distress syndrome. N Engl J Med 338(6):347-354

3. Amato MB et al (2015) Driving pressure and survival in the acute respiratory distress syndrome. N Engl J Med 372(8):747-755

4. Gattinoni L et al (1987) Pressure-volume curve of total respiratory system in acute respiratory failure: computed tomographic scan study. Am Rev Respir Dis 136(3):730-736

5. Gattinoni L, Pesenti A (2005) The concept of "baby lung". Intensive Care Med 31(6):776-784

6. Hickling KG, Henderson SJ, Jackson R (1990) Low mortality associated with low volume pressure limited ventilation with permissive hypercapnia in severe adult respiratory distress syndrome. Intensive Care Med 16(6):372-377

7. Cressoni M et al (2008) Anatomical and functional intrapulmonary shunt in acute respiratory distress syndrome. Crit Care Med 36(3):669-675

8. Cressoni M et al (2014) Lung inhomogeneity in patients with acute respiratory distress syndrome. Am J Respir Crit Care Med 189(2):149-158

9. Fan E et al (2016) Venovenous extracorporeal membrane oxygenation for acute respiratory failure: a clinical review from an international group of experts. Intensive Care Med 42(5):712-724. doi:10.1007/ s00134-016-4314-7

10. Gattinoni L et al (2016) The "baby lung" became an adult. Intensive Care Med 42(5):663-673 